\title{
Diagnosis
}

\section{Tachypnea is a useful predictor of pneumonia in children with acute respiratory infection}

Palafox M, Guiscafré H, Reyes H, et al. Diagnostic value of tachypnoea in pneumonia defined radiologically. Arch Dis Child $2000 \mathrm{Jan} ; 82: 41-5$.

QUESTIONS: In children with acute respiratory infection, is tachypnea accurate for detecting pneumonia? Does disease duration, age of the child, or presence of malnutrition influence sensitivity and specificity?

Design

Blinded comparison of respiratory rate with findings on chest radiography.

Setting

A state referral hospital in Tlaxcala, Mexico.

\section{Participants}

110 children who were 3 days to 5 years of age (55\% were $\geqslant 1 \mathrm{y}$ of age) and had acute respiratory infection. Children who were clinically diagnosed with pneumonia were matched to control children with acute respiratory infection. 30\% of children were underweight. Exclusion criteria were chronic diseases, genetic abnormalities, neurological diseases, bronchial asthma, or septicaemia.

Description of test and diagnostic standard A paediatrician measured the respiratory rate by observing the child's chest movements for 1 minute while the child rested with no crying or fever. Tachypnea was defined as a respiratory rate $>60$ breaths/minute in children $<2$ months of age, $>50$ breaths/minute in

Source of funding:

no external funding.

For correspondence:

Dr H Martínez,

Research Unit on

Epidemiology and

Health Services,

Mexican Social

Security Institute,

Centro Médico

Nacional Siglo XXI,

Unidad de Congresos,

Bloque B, 4o Piso,

Avenida Cuauhtémoc

330, Col Doctores,

Mexico 06725 DFF Fax

$+5257610952$

*Abbreviations defined in glossary; LRs calculated from data in article. children 2 to 12 months of age, and $>40$ breaths/ minute in children $\geqslant 1$ year of age. The diagnostic standard was chest radiography (not clinical diagnosis).

\section{Main outcome measures}

Sensitivity and specificity for detecting pneumonia.

\section{Main results}

59 children (54\%) had a clinical diagnosis of pneumonia, but only 35 children (32\%) had findings on radiology. The table shows the sensitivity, specificity, and likelihood ratios. Sensitivity and specificity were lowest in children with a disease duration of $<3$ days $(\mathrm{p}<0.01)$ (table); age or presence of malnutrition did not influence results.

\section{Conclusions}

In children with acute respiratory infection, tachypnea had a sensitivity of $74 \%$ and specificity of $67 \%$ for detecting pneumonia but did not perform as well when disease duration was $<3$ days. Age or presence of malnutrition did not influence results.

Test characteristics of tachypnea for detecting pneumonia in children with acute respiratory infection*

\begin{tabular}{llllll} 
Test & Patient group & Sensitivity (95\% Cl) & Specificity (Cl) & +LR & -LR \\
Tachypnea & All children & $74 \%(60$ to 88$)$ & $67 \%$ (56 to 77$)$ & 2.2 & 0.4 \\
\hline & Disease duration <3 days & $55 \%(40$ to 72$)$ & $64 \%(49$ to 80$)$ & 1.5 & 0.7 \\
\hline & Disease duration 3 to 5 days & $64 \%(49$ to 78$)$ & $66 \%(51$ to 80$)$ & 1.9 & 0.5 \\
\hline & Disease duration $\geqslant 6$ days & $93 \%(84$ to 102$)$ & $73 \%(57$ to 89$)$ & 3.4 & 0.1 \\
\hline Clinical judgment & All children & $74 \%(61$ to 88$)$ & $56 \%(48$ to 69$)$ & 1.7 & 0.5 \\
\hline
\end{tabular}

\section{COMMENTARY}

Many clinicians currently consider pulse oximetry to be a vital sign. In contrast, the studies by Palafox and Rajesh and their colleagues from developing nations emphasise the importance of an accurately measured respiratory rate. Palafox $e t$ al studied young children in Mexico. They selected children with clinically diagnosed pneumonia and an equal number of children with other acute respiratory illnesses, ensuring a sample with a high prevalence of radiographically proven pneumonia (32\%). The radiographic determination of pneumonia was the reference standard to which the finding of tachypnea was compared.

Tachypnea, defined according to World Health Organisation (WHO) recommendations, was present in $74 \%$ of children with pneumonia and in $33 \%$ of those without pneumonia. The presence of tachypnea approximately doubled the odds of pneumonia, and its absence decreased the odds by about half. These findings are similar to those of a systematic literature review on the diagnosis of pneumonia in infants in which the authors concluded that tachypnea was the best single finding for ruling out pneumonia. In that review, likelihood ratios for pneumonia in the presence of tachypnea ( + LR) ranged from 1.6 to 3.2 with the exception of infants $<2$ months of age.

Likelihood ratios for pneumonia when tachypnea was not present (-LR) ranged from 0.3 to 0.8 . The unique finding of Palafox $e t$ al was that, as hypothesised, the finding of tachypnea was less sensitive and less specific in infants and children who had been sick for $<3$ days.

Rajesh et al in India found tachypnea to be a similarly useful marker for hypoxia in sick infants $<2$ months of age. A cut off point of 60 breaths/minute had the best combination of sensitivity and specificity in this age group (in agreement with the WHO recommendations). Tachypnea was present in $81 \%$ of hypoxic infants and in 32\% of those who were not hypoxic. Thus, tachypnea is sensitive for ruling out hypoxia in young infants, although approximately 1 in 5 hypoxic infants will be missed using tachypnea alone.

The study sample included many severely ill infants; $16 \%$ died. In addition to pneumonia (present in $34 \%)$, septicaemia $(12 \%)$, and meningitis (14\%), several less frequent conditions were found. 